\title{
Practitioners' actions inhibited patient participation in self care decision making
}

Paterson B. Myth of empowerment in chronic illness. J Adv Nurs 2001 Jun;34:574-81

\section{QUESTION: How do people with long standing type 1 diabetes mellitus manage self care decision making and relationships with practitioners?}

Design

Grounded theory, guided by symbolic interactionism.

Setting

Vancouver, British Columbia, Canada.

\section{Participants}

22 Caucasian adults (mean age 43 y, $64 \%$ women) with type 1 diabetes of $\geqslant 15$ years' duration (mean $30 \mathrm{y}$ ) who nominated themselves or were nominated by their physicians as expert self care managers (ie, able to make trustworthy decisions about self management and to maintain good glycaemic control).

Source of funding:

BC Health Research

Foundation.

For correspondence: $\operatorname{Dr} B$

Paterson, School of

Nursing, University of

British Columbia,

Vancouver, British

Columbia, Canada.

paterson@nursing.ubc.ca

\section{Methods}

Data were collected from audiotaped think aloud periods, in which participants described their self care decision making over three 1 week periods; individual interviews held before and after think aloud periods; and a 2 hour focus group interview at the study conclusion. Transcripts of think aloud periods were used to

\section{COMMENTARY}

Through collection of data over three 1 week periods and using audiotaped think aloud periods, interviews, and focus groups, Paterson details well grounded and trustworthy data supportive of client perceptions of disempowerment in their relationships with practitioners. Paterson's study shows that healthcare providers, despite acceptance of a social mandate to "enable people to gain control over and to improve their health", ${ }^{1}$ exhibit behaviours that diminish the valuable partnerships between provider and patient.

Paterson does not describe the professional backgrounds of all the "practitioners" referred to in this study, although physicians and a diabetes educator are specified in one or more quotes in the text. However, regardless of specific professional background, all healthcare providers should take note of these study findings. Clearly, practitioners' practices convey messages that contradict the tenets of empowerment. Although they may invite people with chronic illness to engage in participatory decision making, practitioners' behaviours and practices may actually inhibit or negate their intended goal. The author suggests that there is a need for further research about the overt and covert ways that practitioners cling to professional dominance and the ways in which they may impede participatory decision making.

Current concerns related to healthcare financing, the nursing shortage, and the "cost" of time for patient assessment and goal implementation influence nurses' abilities to assist patients to become empowered. Continued research should focus on creative, ethical, and cost-effective strategies to increase support for professional practice that is responsive to patient perceptions of disease and care, and that promote stronger provider-patient partnerships and improved patient health and professional practice.

$$
\text { Carolyn Spence Cagle, RNC, PhD }
$$
Associate Professor College of Health and Human Sciences Texas Christian University Fort Worth, Texas, USA

1 Canadian Public Health Association, Health and Welfare Canada, and the World Health Organization. Ottawa Charter for Health Promotion. International Conference on Health Promotion, Ottawa, Ontario, November 17-21, 1986.

develop interview prompts. All transcripts were analysed using constant comparative techniques.

\section{Main findings}

13 participants indicated that they had experienced collaborative partnerships with practitioners that allowed them to participate actively in decision making about their care. Although practitioners intended to be "empowering", they tended to contradict patient empowerment in 2 ways: by discounting the participants' experiential knowledge and by not providing access to necessary resources for informed decision making.

Participants described episodes in which practitioners invited them to participate in decisions, but then discounted their experiential knowledge. Practitioners communicated their distrust of participants' experiential knowledge by emphasising objective data (eg, laboratory results), quizzing participants about their diabetes knowledge if they suggested changes in regimens, disregarding data that contradicted textbook information (eg, unique bodily cues of hypoglycaemia), and by communicating expectations of compliance, either by blaming patients for high blood glucose concentrations or through excessive monitoring behaviour.

Participants also felt that practitioners contradicted empowerment by not providing the necessary resources (information, time, and monetary) for informed decision making. Participants reported that the way in which information was presented affected their willingness and ability to engage in shared decision making. Use of medical jargon accentuated the power difference between participants and practitioners. Some participants found physician advice to be inappropriate for their life situation (eg, a suggestion to get a treadmill without considering the participant's limited money and space). Time was described in terms of the duration of a patient's relationship with the practitioner, the pace and length of office visits, and the length of waiting time for appointments. Participants felt that changing practitioners or seeing specialists infrequently limited opportunities for participatory decision making. They interpreted tight scheduling of appointments and a practitioner's hurried approach as unwillingness to include patients in decision making. Because of the costs associated with long waiting times for appointments (child care, parking), participants who waited for $>1$ hour to see their practitioner were often reluctant to take the time needed for participatory decision making.

\section{Conclusion}

Patients with long standing type 1 diabetes described how practitioners, although verbalising their support of participatory decision making, often acted in ways that prevented such participation. 